\section{GP289 KIDNEY INVOLVEMENT IN TUBEROUS SCLEROSIS COMPLEX}

Sultan Ceren Yıldırım, Gülen Gül Mert, Aysun Karabay Bayazı**, Şakir Altunbaşak. Çukurova University, Adana, Turkey

\subsection{6/archdischild-2019-epa.348}

Purpose Tuberous sclerosis complex (TSC) is an inherited neurocutaneous disorder that is characterized by pleomorphic features involving many organ systems. Renal manifestations are the second most significant cause of morbidity and mortality in patients with tuberous sclerosis complex (TSC), and include renal cysts, angiomyolipomas (AMLs) and malignant tumors. In this study, we investigated patients with tuberous sclerosis in our clinic for renal involvement.

Methods In our clinic, the renal manifestations of children with TSC between $0-18$ years of age were evaluated. Age of the first diagnosis, TSC history of family, renal ultrasonography findings of angiomyolipomas and cysts such as size, quantity, location, renal function tests, urinalysis, presence of hypertension, additional organ involvement,and the presence of renal cell carcinoma were assessed.

Results There were 17 (8 male and 9 female) patients with TSC. The mean age of the patients was 11.6 years and first diagnosis time was 2,3 years. Angiomyolipoma was the most frequent lesion (15 of 17 patients) and twelve of them were bilateral. At the time of diagnosis 3 patients had angiomyolipomas. The sizes of AMLs of the patients were smaller than $5 \mathrm{~cm}$. Six patients had also renal cysts and 2 of them with renal cysts had no AML. Additional organ involvement was observed in 3 patients. All of the patients had normal renal function tests and urinalysis.

Conclusion The most common renal lesions in TSC are angiomyolipomas and kidney cysts. At the time of TSC diagnosis, all the children must be screened for renal involvement and changing of renal findings in TSC with time should not be forgetten since the new findings can be added to old ones.

\section{GP290 CAN WE RELY ON PYURIA TO DIAGNOSE URINARY TRACT INFECTION IN CHILDREN?}

${ }^{1}$ Jennifer Cox*, ${ }^{1} \mathrm{M}$ Kenosi, ${ }^{1}$ Laura Whitla, ${ }^{1,2}$ Montaseur Nadeem. ${ }^{1}$ Tallaght University Hospital, Dublin, Ireland; ${ }^{2}$ Trinity College, Dublin, Ireland

\subsection{6/archdischild-2019-epa.349}

Urinary Tract Infections (UTI) occurs in up to 7\% of febrile infants and young children (1). The most common uropathogen is Escherichia coli (E.coli) (2). Presence of pyuria $(\geq 10$ white blood cells per cubic millimetre $\left(\geq 10 \mathrm{WBC} / \mathrm{mm}^{3}\right)$ on urinalysis is essential to diagnose UTI (2). We set out to examine whether there is a link between the absence of pyuria and a positive urine culture for a known uropathogen.

This was a retrospective cohort study of 248 patients admitted with symptomatic UTI between January 2015 and December 2016. Mean (SD) age was 31.27 (42.54) months and $101(40.7 \%)$ patients were male. Of 248 patients with UTI, E. coli was documented as the causative pathogen in 221 cases $(89.1 \%)$, Klebsiella species in 8 (3.2\%), Proteus species in $8(3.2 \%)$, Pseudomonas species in 7 (2.8\%), Enterococcus species in $3(1.2 \%)$ and Group B streptococcus in 1 $(0.4 \%)$. All patients with UTI due to E.coli infection exhibited
$>10 \mathrm{WBC} / \mathrm{mm}^{3}$ on urinalysis (p value 0.001 ). However, in those with UTI caused by non-E.coli uropathogens, $<10$ $\mathrm{WBC} / \mathrm{mm}^{3}$ on urinalysis was documented in two of eight (25\%) patients with UTI secondary to Proteus infection, two of eight (25\%) individuals with Klebsiella UTI, one of seven (14.3\%) patients with UTI due to Pseudomonas infection and one of three $(33.3 \%)$ patients with UTI caused by Enterococcus species.

In conclusion, 9 in 10 patients with UTI experienced E.coli uropathogen on urine culture. Pyuria with $>10 \mathrm{WBC} / \mathrm{mm}^{3}$ on urinalysis was documented in all patients with E.coli uropathogen. However approximately one fifth of patients with UTI due to non-E.Coli uropathogens experienced absence of pyuria on urinalysis. Considering the possibility of absence of pyuria in those with UTI due to non-E.coli uropathogens, using reliable adjunctive biomarkers in diagnosis UTI while waiting urine culture need to be explored through further research.

\section{REFERENCES}

1. Shaikh N, Morone NE, Bost JE, Farrell MH. Prevalence of urinary tract infection in childhood: a meta-analysis. Pediatr Infect Dis J 2008;27:302-8.

2. Shaikh N, Shope TR, Hoberman A, Vigliotti A, Kurs-Lasky M, Martin JM. Association Between Uropathogen and Pyuria. Pediatrics 2016;138.

\section{GP291 AUDIT OF THE IRISH NATIONAL CENTRE FOR PAEDIATRIC RHEUMATOLOGY NEW PATIENT WAITING LIST}

Edel Carberny*, Derek Deely, Emma Jane Mac Dermott, Orla Killeen. Our Lady's Childrens Hospital Crumlin, Dublin, Ireland

\subsection{6/archdischild-2019-epa.350}

Introduction Juvenile idiopathic Arthritis (JIA) is the most common inflammatory disorder of childhood. Early recognition and optimal treatment of JIA is associated with reduced mortality and morbidity. Wait times for new patients to be reviewed by paediatric rheumatologists in Ireland are significantly outside the Standards of Care for children and young people with JIA (2010). These recommend that patients with suspected JIA be seen by a paediatric rheumatologist within 42 days of the referral being made.

Advanced practice physiotherapist (APP) provided triage clinics have successfully reduced waiting lists and provided intermediate care pathways for patients who do not necessarily require rheumatologist review (Stanhope et al., 2012). This service model may be an option to manage waiting lists.

New referrals to the paediatric rheumatology are triaged by rheumatologists based on the information provided in the paper referral as 'urgent', 'soon' and 'routine' as per clinical indications. Those referrals categorised as urgent require consultant review, however it may be possible for referrals in the 'routine' and 'soon' categories to be reviewed by an APP.

Method The active waiting list for new referrals categorised as 'soon' and 'routine' were audited in July 2018 to identify the characteristics of referrals and determine the number of referrals that would be appropriate for an APP clinic. Referrals for a musculoskeletal (MSK) presentation were deemed appropriate for APP clinic where;

- it appears likely to be a non-inflammatory source of the MSK issue;

- is it was not clear from the paper referral whether an inflammatory joint condition is the source of MSK issue; 
- there is no indication of connective tissue disorder, specific rheumatologic disorder, unexplained and/or significant comorbid medical symptoms, nor of complex neuro-disability history.

Results There are 421 and 437 children in total on the 'routine' and 'soon' waiting lists respectively. These children are waiting on average 631 and 592 days, and the longest wait is 1550 and 1516 days on the 'routine' and 'soon' waiting lists respectively. Review of the diagnosis provided on the referral indicates that $89 \%$ on the 'routine' waiting list and $65 \%$ on the 'soon' list are appropriate for APP triage.

Conclusion An APP triage clinic is feasible to help manage the waiting list for paediatric rheumatology. The implementation of an APP triage clinic can ensure that only those referrals that require a consultant review are seen at consultant clinic. All others may be successfully managed by an APP.

\section{GP292 PROFESSIONALS' CONSULTATION SERVICE IN THE CHILD SEXUAL ABUSE (CSA) UNIT}

Aideen Walsh*, Leanne Gregory, Vanessa Hendrick, Eimear Gilchrist, Carol Sheridan. Children's Health Ireland at Crumlin, Dublin, Ireland

\subsection{6/archdischild-2019-epa.351}

Introduction/Background St Louise's Unit provides assessment and therapy services for children/young people where CSA is a concern. The multidisciplinary, multiagency professionals' consultation model was introduced in 2016, and adapted in 2018. Some children/young people referred to the unit for assessment and/or therapy regarding CSA, have other complexities in their lives such as concerns of other types of abuse, living in care and other health concerns (including mental health concerns). Therefore introducing additional specialist CSA services to the child's life, may in fact add to these complexities.

Aims and objectives When a referral is received in St Louise's Unit, the Consultation service aims to bring together the professionals already involved in the child/young person's life by offering additional support for those professionals.

Methodology The interdisciplinary team came together as part of the Children's Health Ireland Quality Improvement and Patient Safety Programme in 2018. A retrospective review of the referrals to St Louise's Unit was carried out and data from referrals from June 2018 to March 2019 was collected.

Results For that 9 month timeframe, there were 89 referrals to St Louise's Unit, with 20 directly to the Consultation Service. Consultation appointments were offered on Wednesday afternoons. Interdisciplinary representation from $\mathrm{St}$ Louise's Unit, Laurels Clinic, Tusla, An Garda Síochána other agencies involved in the child/young person's life all met in St Louise's Unit for a 2 hour meeting. The outcomes were $50 \%$ of referrals were discharged, $43 \%$ were forwarded for assessment and $7 \%$ were referred to therapy services in St Louise's Unit.

Discussion The consultation service is a collaborative and reflective process that allows for shared thinking in complex cases and supports case progression or resolution. Some of the outcomes included working through assessment versus therapy dilemmas with the system around the child; successfully resolving CSA concerns in custody and access cases in a way that was least intrusive for a child; identifying mental health concerns; and preserving foster placement and stability for a child.

Conclusions This structured consultation service in St Louise's Unit has led to collaborative working within the unit and with other professionals involved in the child/young person's life where CSA is a concern. In the 20 cases reviewed, this service supported the professionals already involved in the complexities of the child's life with least intrusion for the child.

\section{GP293 DEVELOPING MARSIPAN INTEGRATED CARE PATHWAYS FOR CHILDREN AND ADOLESCENTS WITH EATING DISORDERS- REAL WORLD CHALLENGES AND RECOMMENDATIONS}

${ }^{1}$ Michael Carter*, 'Aisling Garvey, ${ }^{1,2}$ Louise Gibson, ${ }^{3,4}$ Sara McDevitt. ${ }^{1}$ Dept of Paediatrics, Cork University Hospital, Cork, Ireland; ${ }^{2}$ Dept of Paediatrics, University College Cork, Cork, Ireland; ${ }^{3}$ HSE National Clinical Programme for Eating Disorders, Cork, Ireland; ${ }^{4}$ Dept of Psychiatry University College Cork, Cork, Ireland

\subsection{6/archdischild-2019-epa.352}

Background Anorexia Nervosa has the highest rate of mortality and morbidity of the mental health conditions. Much of this is related to the physical consequences of starvation and can lead to medical admissions. Integrated care pathways have the potential to decrease admission rates, reduce duration of stay, and prevent patients slipping through gaps between services. The Junior MARSIPAN risk assessment guideline is a best practice framework that has potential to enhance integrated decision making between mental health, paediatric and primary care for children and adolescents with eating disorders.

Objective To explore the use of Junior MARSIPAN risk guidelines in collaborative decision making in a Regional Tertiary Referral Hospital, and to understand any associated barriers and enabling factors in their implementation.

Methods Patients discharged from Cork University Hospital with a diagnosis of Anorexia Nervosa/Eating Disorder were identified using HIPE data, over a 2-year time period. Variables collected included: admission and subsequent number of bloods performed, assessment of cardiovascular stability, contact with Child and Adolescent Mental Health Service (CAMHS), MDT meetings, the presence of psychiatric co-morbidities and evidence of discharge planning.

Results Of the 38 patients who presented to hospital, 20 were identified as having been admitted overnight over the time period, involving 16 patients. The mean number of admissions per patient was 4(2-11), mean age 15.1years (9.6-17.8 y) and 14 were female. The majority of admissions $(68 \%)$ were unplanned and most were self-referrals. A total of 11 physicians were involved in the care in addition to 6 CAMHS teams and 2 GPs. There was inadequate evidence of documentation of physical risk parameters (BMI\%, Orthostatic BP), biochemistry for monitoring of refeeding syndrome, and of multidisciplinary approach to care and discharge planning.

Conclusions Complexity and delayed communication was a significant barrier to the implementation of junior MARSIPAN across the 17 different teams involved. There is a need to develop MARSIPAN Lead clinician 'champions' and to standardised approaches across services, adult, Paediatric and child psychiatry, in order to minimise variability in patient clinical care experience. An integrated care pathway, based on the 